Accepted for publication in International Journal of Manpower.

Please cite as: Van Borm, H., Baert, S. (in press): What Drives Hiring Discrimination Against Transgenders? International Journal of Manpower.

\title{
What Drives Hiring Discrimination Against Transgenders?
}

\author{
By Hannah Van Borm, ${ }^{1}$ and Stijn Baert ${ }^{2}$
}

\begin{abstract}
We explore the mechanisms underlying hiring discrimination against transgender women. To this end, we conduct a scenario experiment in which fictitious hiring decisions are made about transgender or cisgender female job candidates. In addition, these candidates are scored on statements related to theoretical reasons for hiring discrimination given in the literature. The resulting data are analysed by means of a multiple mediation model. Our results suggest that prejudices with respect to the health of transgender individuals mediate unfavourable treatment of them. However, this mechanism is compensated by a beneficial perception concerning transgender women's autonomy and assertiveness.

Keywords: transgender women, taste-based discrimination, statistical discrimination, hiring, scenario experiment, mediation analysis.

JEL: J15, J71, J16, J24, J23.
\end{abstract}

\footnotetext{
${ }^{*}$ Acknowledgements. We are grateful to Jens Agerström, Vojtech Bartos, lan Burn, Gunn Elisabeth Birkelund, and Dan-Olof Rooth for their comments which helped us to improve this study substantially.

${ }^{1}$ Corresponding author. Ghent University. Sint-Pietersplein 6, 9000 Ghent, Belgium. +32476807480

${ }^{2}$ Ghent University, University of Antwerp, Université catholique de Louvain, IZA, GLO, and IMISCOE.
} 


\section{Introduction}

Survey research conducted in Belgium, the United Kingdom and the United States shows that substantial numbers of transgender individuals perceive discrimination as a struggle for their labour market career. More concretely, they feel unfavourable treated in terms of hiring chances (Badgett et al., 2007; FRA, 2014; Grant et al., 2011; James et al., 2016; Motmans et al., 2009; Valfort, 2017) as well as wage outcomes (Schilt and Wiswall, 2008; Valfort, 2017), promotion opportunities (Badgett et al., 2007; Grant et al., 2011; James et al., 2016; Motmans et al., 2009), and dismissal decisions (Badgett et al., 2007; Budge et al., 2010; Grant et al., 2011; James et al., 2016; Motmans et al., 2009; Schilt and Wiswall, 2008). Moreover, this self-reported unfavourable treatment seems to be of a larger extent than similar forms of discrimination against sexual minorities (gay men and lesbian women in particular). Interestingly, the reported levels of self-reported unfavourable treatment are higher among transgender women, on which we will focus in this study, than among transgender men (Grant et al., 2011). ${ }^{1}$

From a theoretical point of view, there are several reasons why employers may indeed discriminate against transgender individuals. This unfavourable treatment is consistent with the leading economic discrimination theories, i.e. Becker's (1957) model of taste-based discrimination and Arrow's (1973) model of statistical discrimination. The distaste to collaborate with transgender individuals in respect of employers, co-workers, and customers, may relate to the well-documented phenomenon of transphobia, i.e. emotional disgust relative to people who do not meet social expectations about gender (Hill and Willoughby, 2005). Statistical discrimination may be particularly rooted in the health-related stigma of transgender individuals. In this respect, transgender individuals are related to higher risks of mental problems (and suicide) and HIV in the literature (Drydakis, 2017). However, this statistical discrimination might be erroneous in a sense that it may be based on flawed assumptions for particular groups of transgender individuals. Indeed, the risk of health problems for transgender individuals is found (i) to vary across the different stages of transitioning (Brewster et al., 2014; Drydakis, 2017) and (ii) to depend on the extent to which transgender individuals manage to be socially perceived as male or female (Hughto et al., 2015). On the other hand, for transgender women, two documented stereotypical 
mechanisms may result in positive statistical discrimination (compared to cisgender women). First, as transgender women were male before, they are shown to be ascribed particular male characteristics that may adhere to the ideal of masculinity associated with labour market success (Baert, 2014; Schilt and Wiswall, 2008). In particular, transgender women seem to be ascribed higher levels of autonomy and assertiveness. Second, transgender women are expected to give birth to fewer children than cisgender women, ${ }^{2}$ and, as a consequence, may avoid the well-documented penalty of motherhood in the labour market (Baert, 2014).

While during the last decade research using experimental and quasi-experimental methods to measure labour market discrimination against sexual minorities exploded (Baert, 2014; Baert, 2017; Baert, in press; Drydakis, 2014; Drydakis, 2015; Patacchini et al., 2015; Weichselbaumer, 2015), corresponding attempts to objectively measure discrimination against transgender individuals in the labour market are scarce. More concretely, we are aware of four studies in this respect. First, Bardales (2013) and Make The Road New York (2010) conducted audit studies. In the former study, fictitious written job applications from female candidates were sent to 109 vacancies in San Antonio and Houston. Revealing the membership of two transgender organisations, which was randomly added to these applications, lowered positive call-back by $35.1 \%$. In the latter study, matched pairs of (male or female) job applicants, one transgender and one control, applied for jobs in New York-the transgender candidates explicitly informed employers of their transgender status. In only two of the 24 tested vacancies the transgender applicant got a job offer, while their control counterparts got 12 offers. Second, Carpenter et al. (2016) and Geijtenbeek and Plug (2015) used large population-based survey data from the United States and administrative data from the Netherlands, respectively, to estimate wage penalties for transgender individuals. Their estimates reveal employment and wage penalties for being transgender and are, thereby, consistent with labour market discrimination based on this characteristic. ${ }^{3}$

In this article, we take the logical next step in this literature on labour market discrimination against transgender individuals. More concretely, we explore the mechanisms underlying this established discrimination. To this end, we present a scenario experiment in the sense of Baert and De Pauw (2014) and Van Hoye and Lievens (2003). 
Participants to this experiment make fictitious hiring decisions with respect to a (transgender or cisgender) female job candidate. In addition, the participants evaluate their candidate concerning statements related to the aforementioned theoretical reasons for discrimination. This allows us to explore these theories' relative empirical importance. In addition, as we randomise between various sorts of jobs and as we survey our participants on several characteristics, we are able to provide suggestive evidence on how transgender discrimination is heterogeneous by these job and recruiter characteristics (in particular, male-dominated versus female dominated occupations, conformity to classical gender roles, and contact with transgender individuals).

Besides its academic contribution, the present study has a clear societal relevance. Identifying discrimination, as done by the reviewed studies, is one thing; tackling it is another. Clearly, targeted policy measures are needed given the substantial labour market discrimination against transgender individuals-transgender women in particularmeasured. However, to combat this discrimination effectively, one needs to understand its driving factors. More concretely, to design adequate policy actions one has to gain insights into why employers discriminate against transgender individuals-the mechanisms underlying this discrimination-and in which situations this discrimination is particularly high-its moderators. This study provides a first exploration of these mechanisms and moderators.

\section{Data}

\subsection{Participant Selection}

We conducted our experiment in October and November 2016. In preparation of this experiment, we listed all vocationally-oriented Bachelor programs and academicallyoriented Master programs related to human resource management at universities and university colleges in the province of Antwerp, in Flanders (Belgium). In total, we found 12 such programs. For these programs, we contacted the lecturers of the obligatory courses of the first semester of the last year of these programs. We asked them whether we could 
conduct our experiment with their students at the start of one of their classes.

In the end, we were able to conduct our scenario experiment with 310 last-year students, enrolled in 10 programs related to human resource management. This experiment was conducted by means of a paper-and-pencil survey comprising a booklet containing experimental instructions and a post-experimental survey. The participants were instructed to first fill in the former file and then the latter one (without returning to the booklet with experimental instructions).

\subsection{Experiment}

At the beginning of the booklet with experimental instructions, the participants were informed about their role of recruiter for a firm selling building materials. In this role, they were engaged in filling a vacancy in one out of three occupations: management assistant, logistics clerk, or site manager at a building yard. These jobs were chosen as, following information provided by the Public Employment Agency of Flanders (PEAF), the first occupation is female-dominated, the second has a mixed gender representation, and the last is male-dominated. ${ }^{4}$ The participants were briefed about the content of the job they had to fill and the desired profile for the job. This information, based on examples from the vacancy database of the Public Employment Agency of Flanders, is summarised in Table A.1 (in the Online Appendix).

Then, the participants had to screen one résumé of a candidate with adequate qualifications for the job. This candidate was female and had five years of experience in the same occupation as the one for which she applied. The candidate applying for a job as a management assistant (logistics clerk) ((site manager)) held a Bachelor in Office Management (Logistics Management) ((Construction)). In addition, the résumé revealed the following characteristics: (i) born and living in the city of Ghent; (ii) Belgian nationality; (iii) living single; (iv) excellent computer skills (office applications); (v) excellent Dutch, very good English and French and good German language skills; and (vi) being stress proof, communicative, well-organised, punctual, and a team-player.

Besides alternating between three occupations (and related profiles of candidates), we also alternated between transgender and cisgender candidates. So, transgender and 
cisgender candidates were randomly assigned to the participants of the experiment, with a probability of 0.50. As advised by Transgender Infopunt, a Flemish organisation guiding transgender individuals, the transgender identity was disclosed as follows. Firstly, after the name of the candidate, it was mentioned that she was born with a male name. So, while the control candidate just mentioned her name (the typically Flemish and female sounding name 'Sarah Mertens'), for the transgender candidate an additional line with '(born as Tom Mertens)' was added. Secondly, with respect to the gender of this candidate 'Female gender (transgender)' (instead of 'Female gender' for the control candidate) was mentioned. As a consequence of this design, the 'transgender' candidate in our experiment was actually 'openly transgender' and the 'cisgender' candidate was actually a candidate with an unrevealed status in this respect. We return to this point in Section 4.

In Belgium, transgender individuals can change their official first name and gender by going through two separate processes. ${ }^{5}$ Both of these processes changed by January 2018, i.e. after our experiment was conducted. Before January 2018, the application for a first name change could be submitted as soon as one started with hormone replacement therapy, i.e. the introduction of hormones associated with the gender that the patient identifies with. With respect to the application for a change in official gender, before January 2018, a gender change could only be approved in case (i) the transgender person had the constant and irreversible inner conviction to belong to the opposite sex than that stated in her/his birth certificate, (ii) this person had undergone a gender reassignment surgery that brought her/him into line with that opposite sex, and (iii) this person was no longer able to conceive children in accordance with the earlier sex. Since January 2018, both concerning name and gender change, only a statement on honour is needed in which it is written down that one's gender in the birth certificate does not correspond to one's experienced gender identity. So, the legislation with respect to gender change at the moment of our experiment asked for treatments many transgender individuals could not or did not want to undergo, while name change could be approved on less stringent conditions. As a consequence, many transgender individuals have gone through life with their original male/female gender, but with an official female/name (Motmans et al., 2009). Therefore, an alternative to how we disclosed a transgender identity could have been to present a candidate with a male name in combination with a female gender. However, pilot testing, in which we implemented this approach, showed that such candidates were perceived as being odd and/or fake. 
Based on this information, the participants of the experiment had to evaluate their candidate with respect to two clusters of items. First, they were asked to state their general intention to hire the candidate. More concretely, in line with Baert (in press), Di Stasio (2014), and Van Hoye and Lievens (2003), they had to rate two statements: (i) 'I will invite the candidate for a job interview' and (ii) 'I will hire the job candidate' on a 7-point Likert scale going from 'very unlikely' (score 1) to 'very likely' (score 7). In what follows, we will refer to these scores as the 'interview probability' and 'hiring probability' of the candidate, respectively. Second, the participants were asked to share their agreement with statements related to the potential reasons for labour market discrimination against transgender women mentioned in Section 1. With respect to potential taste-based discrimination, we relied on statements used in Baert and De Pauw (2014). That is, the participants had to rate the statements (iii) 'As an employer, I will enjoy collaborating with this candidate' (employer discrimination), (iv) 'My co-workers will enjoy collaborating with this candidate' (employee discrimination), and ( $\mathrm{v}$ ) 'My customers will enjoy collaborating with this candidate' (customer discrimination). With respect to potential statistical discriminated rooted in productivity-related prejudices concerning the autonomy, assertiveness, likelihood of maternity leave, and health of the candidate, we presented the following statements: (vi) 'This candidate will provide sufficient autonomy', (vii) 'This candidate will provide sufficient assertiveness', (viii) 'This candidate will be on maternity leave in the short or medium run', and (ix) 'This candidate will be on sick leave in the short or medium run'. Statements (iii) to (ix) also had to be scored on a 7-point Likert scale.

\subsection{Post-Experimental Survey}

The post-experimental survey was the same for all participants, irrespective of their experimental condition by the (job to be filled and) transgender identity of their candidate. In this post-experimental survey, first, the participants had to complete a manipulation check in which they shared their perception of the transgender identity of the candidate (by means of the statement 'The candidate was born as a woman'). To not give away the aim of the experiment, also statements concerning the residence and ethnic origin of the candidate and the job to be filled were added. Again, these statements were scored on a 7-point Likert scale. 
Second, the social background of the participants was surveyed by means of six items: their gender (female or male), their age, their nationality (Belgian or non-Belgian), the highest education degree of their mother (tertiary education or not), the highest education degree of their father (tertiary education or not), and their own sexual orientation (lesbian, gay, or bisexual sexual orientation; or heterosexual orientation).

As a third component of the post-experimental survey, the participants' belief in traditional gender roles was measured. This was done by means of a shortened version of the Attitudes Toward Women Scale (AWS) of Spence et al. (1973). This scale consists of 24 items to be rated on a 7-point Likert scale concerning the desirable roles and rights of women in different contexts, such as educational and intellectual activities, dating and sexual behaviour, marital relationships, and etiquette. Examples of the items used are 'Women should be more in charge of solving intellectual and social problems' and 'Women are less capable of contributing to the economy compared to men'. Averaging the score for these statements yields a number between 1 and 7 ('conformity to classical gender roles scale').

Fourth, we surveyed the participants with respect to their contact with transgender individuals. To this end, we used the scale of West and Hewstone (2012). This scale comprises four items. For each item, participants have to score their level of contact with transgender individuals, going from 'no contact at all' (score 1) to 'very frequent contact' (score 7), in a different context. The four contexts included are: (i) at school and at work; (ii) in daily, superficial social contacts; (iii) in intimate social situations; and (iv) in all sorts of social situations. Averaging the score for these statements yields a number between 1 and 7 ('contact with transgenders scale').

Finally, the participants' social desirability bias was measured using the 13-item version of the Marlowe-Crowne Social Desirability Scale (MC-SDS) developed by Reynolds (1982). This is one of the most used instruments for measuring social desirability (Baert, in press; Beretvas et al., 2002; Sârbescu et al., 2012). Its items express a behaviour that is culturally approved or sanctioned (e.g., 'I sometimes feel resentful when I don't get my way') and participants have to indicate whether these statements apply to them (score 1) or not (score 0). Summing up the scores for these statements yields a total score of social desirable answering between 0 and 13. In our application, we divided this number by 13 to get a 
number between 0 and 1 ('social desirability bias scale').

\subsection{Data Description}

Table 1 describes the experimentally gathered data. More concretely, this table presents summary statistics with respect to the manipulation check (Panel A), occupation and participant characteristics (Panel B and Panel C), and candidate evaluation scores (Panel D), for the two experimental conditions (i.e. the subsamples of participants classified by the transgender identity of their assigned job candidate).

\section{$<$ Table 1 about here>}

Clearly, our (main) experimental manipulation worked. While the average score for the statement 'The candidate was born as a woman' is 6.287 among the participants screening a control candidate, it is only 1.619 among the participants screening a transgender woman. This difference is highly significantly different from 0 ( $t$-value of 30.232). Next, Panel B shows that also the randomisation of the three sorts of vacancies by occupation over the participants (in the two experimental conditions) was successful. The six potential combinations of three occupations and two transgender identities are evenly represented in the sample. The same is true with respect to the randomisation of the transgender identity over the participants of the experiment (Panel C). That is, the participants in the two experimental conditions are very similar in terms of social background, conformity to classical gender roles, contact with transgender individuals, and social desirability bias.

Concerning the internal consistency of the scales included in Panel C, Cronbach's alphacoefficient is 0.848 for the conformity to classical gender roles, 0.693 for the contact with transgenders scale, and 0.640 for the social desirability bias scale.

Finally, Panel D shows that, on average, the interview probability does not vary by the transgender identity of the candidates. Moreover, with respect to the hiring probability, we even find a weakly significantly higher score for the candidates revealing a transgender identity. On the one hand, this non-negative effect contrasts with the evidence reported in Bardales (2013), Carpenter et al. (2016), Geijtenbeek and Plug (2015), and Make The Road New York (2010) for the Netherlands and the United States. On the other hand, these statistics are consistent with the very tolerant public opinion towards sexual minorities in 
Belgium (Baert, in press; ILGA-Europe, 2017) and the particular finding of zero or positive effects (depending on candidate age) on employment opportunities of revealing a lesbian sexual orientation when applying for a job in Flanders in Baert (2014). In addition, although the participants were not informed about the aim of the experiment, we cannot rule out that the mentioned scores with respect to interview invitation and hiring likelihood are positively affected by socially desirable answering. Therefore, in Table A.2 (in the Online Appendix), we provide the interview and hiring probability statistics (i) after excluding those participants with a score for the social desirability bias scale higher than the full sample mean increased with one standard deviation (Panel A) and (ii) after excluding those participants with a score for the social desirability bias scale higher than the full sample mean (Panel B). However, for both restricted samples of participants, we still get a nonnegative effect of revealing a transgender identity on the hiring decisions made by these participants.

Anyway, the first two rows of Panel D of Table 1 indicate that the various mechanisms for hiring discrimination against transgender individuals explored in this study at most seem to cancel each other out (at least within our research sample). This brings us to the key statistics in the lower rows of Panel $D$, which are the starting point of the next section.

\section{Results}

In this section, we analyse our data to investigate the empirical importance of taste-based discrimination (employer discrimination, employee discrimination, and customer discrimination) and statistical discrimination (prejudices with respect to autonomy, assertiveness, maternity leave, and health) in explaining ('mediating') the employment opportunities of the transgender candidates in our experiment. A significant mediation role (or 'mediation effect') for the candidate evaluations related to these mechanisms ('mediators') is conditional on two things. First, candidates' transgender identity should affect the mediators. Second, these mediators should affect the interview probability and/or hiring probability. In Section 3.1, we explore both conditions for each mediator separately. Then, in Section 3.2, we run a multiple mediation model in which the mediators and hiring 
decisions are explained jointly. This allows us to identify the independent importance of the mediation effects. Finally, in Section 3.3, we compare the relative treatment of the transgender and cisgender candidates in our experiment for various subsamples by the job filled and by the participant characteristics. This allows us to explore the moderating role of these characteristics.

\subsection{Mediators of Hiring Discrimination Against Transgender Women: Bivariate} Analysis

Through the scores for the statement, 'As an employer, I will enjoy collaborating with this candidate', we gauge the ground for employer discrimination in respect of our participants in a direct way. As shown in the third row of Panel D of Table 1, the average score for this statement is comparable between the experimental group (that received a resume with a transgender candidate) and the control group (that received a control candidate). We get even a somewhat higher score for the transgender candidate (i.e. 5.232 versus 5.019). However, the difference between these scores is only weakly significant. This finding is consistent with what we found with respect to both candidates' interview and hiring probability, discussed at the end of Section 2.

However, we get a totally different picture when we monitor the average scores for the statements related to co-worker discrimination and customer discrimination. For these statements, the average score for the cisgender applicant is substantially higher than the score for the transgender applicant. These statistics provide empirical support for two reasons for discrimination against transgender women: the concern that co-workers and customers will prefer collaborating with cisgender individuals.

Next, in line with our expectations based on the literature discussed in Section 1, the participants of our experiment evaluated transgender individuals as being more autonomous and assertive. In addition, as expected, the likelihood of maternity leave is assessed as being lower for transgender women compared with cisgender women. On the other hand, transgender women are also seen as being in worse health.

The discussed associations between these evaluation scores and the transgender identity of the candidate hardly change when the research sample is restricted to 
subsamples with a low social desirability bias. There is, however, one exception to this pattern: the weakly significantly premium of being a transgender candidate on the participants' own taste to collaborate with the candidate disappears when excluding individuals with a high social desirability bias.

Column (1) and Column (2) of the correlation matrix shown in Table A.3 (in the Online Appendix) allow us to check whether diverging scores for the mediators (candidate evaluations) are associated with diverging scores with respect to the interview and hiring probability. Indeed, a more positive perception with respect to own taste, co-workers' taste, and customer's taste for collaboration with a candidate yields better employment opportunities. In addition, as expected, when candidates get a higher rating with respect to autonomy, assertiveness, and health-so, a lower rating with respect to sick leave probability-hiring decisions about their candidacy are more favourable. On the other hand, ratings with respect to maternity leave probability are not significantly associated with these hiring decisions.

Taking these two steps together, we can conclude that a bivariate analysis of our data points in the direction of a substantial negative mediating role for (i) expected distaste to collaborate with transgender women in respect of co-workers and customers and (ii) healthrelated stigma. These mechanisms are, in our sample, compensated by a substantial positive mediating role for perceived autonomy and assertiveness, two typically male characteristics that are found to be ascribed to transgender women.

\subsection{Mediators of Hiring Discrimination Against Transgender Women: Multiple Mediation Model}

As shown in Column (3) to Column (9) of Table A.2, the different candidate evaluation scores are highly correlated. As a consequence, some of the mediation effects discussed in the previous subsection might be spurious associations just picking up other, genuine relations. Therefore, in this subsection, we present a multiple mediation model allowing us to get an insight into the independent mediation effects.

In our mediation model, which is schematised in Figure 1, the transgender identity of a job candidate is associated with the likelihood of a beneficial hiring decision in both a direct 
way and an indirect way via the included mediators. Given the particular high correlation between the statements related to co-worker and customer discrimination, the average value of these statements is included as one 'co-worker and customer taste to collaborate' scale (Cronbach's alpha is 0.850 ). For the same reason, the scores for the statements concerning perceived autonomy and assertiveness are combined as one 'perceived autonomy and assertiveness' scale (Cronbach's alpha is 0.768). The three other mediators included relate to the other (single) evaluation statements (i.e. employer taste to collaborate with the candidate, perceived maternity leave probability, and perceived sick leave probability). The resulting mediation model, estimated following the procedure discussed in Hayes (2013), consists of a system of six linear regression equations. In the first five regression models, the mediators are regressed on an indicator of the candidate's transgender identity. In the last regression model, a hiring outcome variable is regressed on the five mediators and the transgender dummy. In our benchmark model, the hiring outcome variable used is the hiring probability. For ease of interpretation, all included variables except for the transgender dummy are standardised by subtracting their sample mean and dividing the result by their sample standard deviation.

\section{<Figure 1 about here>}

The estimation results for our mediation model can be found in Figure 1 and Table A.4 (in the Online Appendix). The total effect of presenting a job candidate with a transgender identity on the hiring probability is, in line with what was discussed at the end of Section 2, weakly significantly positive $(c=0.197, p=0.083)$. This total effect can be decomposed into a direct association and indirect associations via the mediators. The former association, indicating whether the hiring probability is affected by the candidate's transgender status after controlling for the five included mediators, is not significant $\left(c^{\prime}=0.087, p=0.434\right)$.

More interesting, however, are the estimated indirect associations. These mediation effects are the products of the effect of the transgender dummy on the relevant mediator (i.e. the $a_{i}$ in Figure 1 ) and the association of this mediator with the hiring probability (i.e. the $b_{i}$ in Figure 1).

Not surprisingly given the random assignment of the transgender and control identity to the participants, the effect of the transgender dummy on the mediators is completely in line with the statistics presented in the last rows of Table 1 . Perceived maternity leave is about 
one standard deviation lower for transgender candidates $\left(a_{4}=-1.004, p=0.000\right)$, while the negative effect on co-worker and customer taste $\left(a_{2}=-0.416, p=0.000\right)$, the positive effect on perceived autonomy and assertiveness $\left(a_{3}=0.555, p=0.000\right)$, and the positive effectin a quantitative sense-on perceived sick leave probability $\left(a_{5}=0.416, p=0.000\right)$ are about half as large. Finally, again, a weakly significantly positive effect on employer taste to collaborate is found $\left(a_{1}=0.214, p=0.059\right)$.

On the other hand, with respect to the association of the mediators with the hiring probability, perceived co-worker and customer taste to collaborate with the candidate is not a significant driver of the hiring probability of this candidate after controlling for the other mediators $\left(b_{2}=0.078, p=0.071\right)$. So, only employer taste to collaborate (positive association; $b_{1}=0.214, p=0.001$ ), perceived autonomy and assertiveness (positive association; $b_{3}=0.404, p=0.000$ ), and perceived sick leave probability (negative association; $b_{5}=-0.141, p=0.004$ ) remain as significant drivers.

Multiplying the estimated $a_{i}$ and $b_{i}$ coefficients for each moderator yields two statistically significant mediation effects. First, we find a highly significantly positive mediation via perceived autonomy and assertiveness $\left(a_{3} b_{3}=0.224, p=0.000\right)$. This association is also highly significant in economic terms: this mechanism yields, in our sample, a hiring probability that is $22.4 \%$ of a standard deviation higher for transgender candidates, ceteris paribus. Second, we find a significantly negative mediation via perceived sick leave probability $\left(a_{3} b_{3}=-0.059, p=0.031\right)$. This mediation effect is about four times smaller than the mediation effect via perceived autonomy and assertiveness.

We conducted several robustness checks to test the sensitivity of our results for alternative choices with respect to the specification of our mediation model and the restriction of the sample used. First, we re-estimated our model (i) using the interview probability as an outcome and/or (ii) excluding the individuals with a high social desirability bias. Second, given that, by the construction of our experiment, the transgender identity of the fictitious candidate is orthogonal to the occupation and participant characteristics, in our benchmark model, we did not include the latter variables as controls in the mediation model. In an additional analysis, we included these covariates, in all six of the regression equations. Third, for ease of the interpretation, in our benchmark approach, we treated the hiring probability and mediator variables as continuous while they are in fact ordinal in 
nature. Although treating ordinal variables as continuous is common when they have at least five categories (Detollenaere et al., 2017; Detollenaere et al., in press), we also estimated our mediation model as a system of ordered probit models. However, the results discussed above turned out to be robust to these alternative approaches (of which the estimates are available on request).

\subsection{Moderators of Hiring Discrimination Against Transgender Women}

The fact that we did not find evidence for hiring discrimination against transgender women at the level of our full sample (Section 2.4) does not rule out that unfavourable treatment of transgender candidates is apparent among particular subsamples. In other words, this hiring discrimination may be moderated by the occupation of the vacancy filled and by the participants' characteristics.

In this respect, a relatively higher (lower) hiring probability for the transgender candidate in our experiment can be expected in the male-dominated (female-dominated) occupation of site manager. This would be consistent with the lack of fit model (Heilman, 1983) emphasising the importance of gender stereotypes, gender expectations, and gender types of jobs in hiring decisions. Given the more masculine characteristics attributed to transgender women discussed in Section 1, these women should have higher recruitment opportunities compared to cisgender women in typically male jobs but less high recruitment opportunities in typically female jobs.

Therefore, in Panel A of Table A.5 (in the Online Appendix), we present the difference in hiring probability by transgender identity for three subsamples of our data by occupation of the filled vacancy. Column (5) shows that this difference is very similar for the three occupations. So, we do not find evidence for moderation effects in this respect. A regression analysis in which the hiring probability is regressed on the transgender dummy (see Section 3.2) and interactions between this dummy and occupational dummies, yields the same conclusion.

With respect to moderators at the recruiter side, first, hiring discrimination against transgender women can be expected to be higher among individuals with a higher conformity to classical gender roles. Indeed, transphobia and genderism, as discussed in 
Section 1, are expected to be higher among them (Kerns and Fine, 1994). Second, contact with transgender individuals is expected to break negative stereotypes against them. As a consequence, lower levels of discrimination are expected in respect of recruiters who have more contact with transgender individuals. For this reason and for a feeling of commonality, third, lesbian, gay, and bisexual recruiters may also discriminate less against transgender individuals (Stone, 2009).

In Panel B of Table A.5 we present the difference in hiring probability by transgender status for subsamples of our data by these three potential moderators. As socio-economic background characteristics may correlate with these potential moderators (e.g., males have been found to have a higher conformity to classical gender roles; Kerns and Fine, 1994), we also split our sample by the measured social background characteristics. By doing that, we see that our overall finding of a weakly higher hiring probability for transgender candidates is somewhat driven by the female participants, the participants with a lower educated mother, the heterosexual participants, and the participants with a low conformity to classical gender roles. For these subsamples, weakly significantly differences in hiring probability in favour of transgender candidates, are found. However, further analysis shows that we cannot speak of real moderation by the gender, maternal education level, sexual orientation, or conformity to gender roles of the participants. Indeed, Panel B shows that the difference in hiring probability is also (insignificantly) positive for the male participants, the participants with a higher educated mother, the lesbian, gay, and bisexual participants, and the participants with a high conformity to classical gender roles. Moreover, regressing hiring probability on transgender status and interactions between this status and participants characteristics does not yield significant interactions.

\section{Conclusion}

We presented a scenario experiment in which fictitious hiring decisions were made with respect to transgender or cisgender female job candidates. In addition, evaluations of these candidates related to dominant explanations in the literature for hiring discrimination against transgender individuals were shared. The participants to our experiment did not 
have a distaste to collaborate with transgender workers themselves but at the same time feared a similar distaste in respect of co-workers and customers. In addition, we provided suggestive evidence for health-related stigma of transgender women. On the other hand, the participants to our experiment had prejudices in favour of transgender candidates: they were perceived as more autonomous and assertive and less likely to be on maternity leave in the short or medium run. When modelling these perceptions as competing mediators for the fictitious hiring decisions made, we found that two explanations were dominant, i.e. the negative health-related prejudices and the positive perception of transgender women's autonomy and assertiveness. From a policy perspective, these results indicate that transgender individuals have every interest in including information signalling their good health (e.g., by means of mentioning their sports activities) into their job applications.

This study innovated in being the first to explore the relative empirical importance of dominant explanations for hiring discrimination against transgender women. However, inherent to its explanatory nature in general and its aim to study these explanations jointly, this study is limited in several aspects. For the following reasons, rather than giving its results a structural interpretation, this study should be seen as a starting point for the investigation of the mechanisms underlying labour market discrimination against transgender workers.

First, while our scenario experiment had the advantage that it allowed us to look into multiple mechanisms underlying hiring discrimination against transgender women together, it is limited by its laboratory setting. Although it has been shown that decisions made in scenario experiments highly correlate with actual behaviour (Baert and De Pauw, 2014; Van Belle et al., 2017), it can never be proofed that the dynamics measured in a certain lab experiment will be (of) the same (magnitude) in the field. In particular, participants who are aware that they are taking part in an experiment, might act in a socially desirable way. In our context, this socially desirable answering may have translated into candidate evaluations biased favourably for transgender women. As a consequence, the health-related stigma of transgender women identified may be underestimated and the prejudices in favour of these candidates may be overestimated. However, in this respect, it was reassuring to see that these candidate evaluations for the subsample of participants with a low social desirability bias hardly differed from those for our full sample.

A second limitation is that we did not engage real recruiters as participants for our 
experiments but relied on students. However, many studies suggest that, particularly with respect to evaluating job candidates, students' evaluations do not substantially diverge from those of professional recruiters (Baert, in press; Hosoda et al., 2003; Falk et al., 2013). Moreover, the last-year students in a program related to human resource management engaged can be thought of as tomorrow's recruiters.

Third, we only investigated the mechanisms of interest in the context of (fictitious) hiring decisions about individuals with a specific profile applying for one out of three particular occupations. As a consequence, our results cannot be easily generalised to other settings, with different candidate profiles and occupations. In particular, by means of our design we compare the hiring outcomes between an openly transgender candidate and a control candidate. As a consequence, this comparison also includes the costs associated with disclosing one's transgender identity (and thereby, potentially, the costs associated with a signal of activism; Baert, 2014; Weichselbaumer, 2015). ${ }^{6}$ Therefore, we are in favour of future contributions that investigate moderators of discrimination against transgender individuals at the employee side. Besides heterogeneity in discrimination by openness concerning the transition process, also potential heterogeneity by level of social integration - see our discussion on erroneous statistical discrimination in the introductioncould be investigated.

Fourth, as for all empirical studies, our statistical analyses are limited by the finite size of our research sample. In this respect, our number of participants is comparable to that of other scenario experiments (Baert, in press; Di Stasio, 2014; Van Hoye and Lievens, 2003). Nevertheless, related to our finite sample size, multiple statistics included in our article are only statistically significant at the $10 \%$ level. A larger research sample would have enabled us to provide a more decisive evaluation of the related associations. In particular, we were not able to identify significant heterogeneity in hiring discrimination by the conformity to classical gender roles and level of contact with transgender individuals of the participants of our experiment. Whether this was due to the size (and composition) of our sample or due to the fact that hiring discrimination against transgender women is indeed homogeneous by these characteristics remains an open question.

Fifth, while the effect of revealing a transgender identity on the candidate evaluations (i.e. the $a_{i}$ within our mediation model) can be given a causal interpretation-at least within 
our experimental setting-this is not the case for the estimated associations of these evaluations with the fictitious hiring decisions (i.e. our $b_{i}$ ). Although we attempted to capture the most relevant potential mechanisms underlying hiring discrimination against transgender women, it is still possible that the included mediators correlate with other, unobserved, prejudices. As a consequence, also our mediation effects (i.e. our $a_{i} b_{i}$ ) should be seen as associations rather than as causal effects. Measuring causal mediation effects could only be realised when also our mediators would be experimentally manipulated, which was not feasible within our context (and does not seem feasible within a setting in which multiple mechanisms are jointly investigated, as we aimed).

\section{References}

Arrow, K. J. (1973), "The theory of discrimination", in Ashenfelter, O., Rees, A. (Eds.), Discrimination in Labor Markets, Princeton University Press, Princeton.

Baert, S. (2014), "Career lesbians. Getting hired for not having kids?", Industrial Relations Journal, Vol. 45 No.6, pp. 543-561.

Baert, S. (2017), "Hiring Discrimination: An Overview of (Almost) All Correspondence Experiments Since 2005", IZA Discussion Paper Series [10738], Institute of Labor Economics, Bonn.

Baert, S. (in press), "Hiring a Homosexual, Taking a Risk? A Lab Experiment on Employment Discrimination and Risk Aversion", Journal of Homosexuality. DOI: 10.1080/00918369.2017.1364950.

Baert, S. and De Pauw, A.-S. (2014), "Is ethnic discrimination due to distaste or statistics?", Economics Letters, Vol. 125 No. 2, pp. 270-273.

Bardales, N. (2013), "Finding a job in "a beard and a dress". Evaluating the effectiveness of transgender anti-discrimination laws", Unpublished Manuscript.

Becker, G. S. (1957), The Economics of Discrimination, University of Chicago Press, Chicago 
Brewster, M. E., Velez, B., De Blaere, C. and Moradi, B. (2012), “Transgender individuals' workplace experiences: The applicability of sexual minority measures and models", Journal of Counseling Psychology, Vol. 59 No. 1, pp. 60-70

Brewster, M. E., Velez, B. L., Mennicke, A. and Tebbe, E. (2014), "Voices from beyond: A thematic content analysis of transgender employees' workplace experiences", Psychology of Sexual Orientation and Gender Diversity, Vol. 1 No. 2, pp. 159-169.

Carpenter, C. S., Eppink, S. T. and Gonzales, G. (2016): "Transgender status, employment, and income", Unpublished Manuscript.

De Sutter, P. (2001), "Gender reassignment and assisted reproduction. Present and future reproductive options for transgender people", Human Reproduction, Vol. 16 No. 4, pp. 612-614.

Detollenaere, J., Willems, S., Baert, S. (2017), "Volunteering, income and health", PLOS ONE, Vol. 12 No. 3, e0173139.

Detollenaere, J., Willems, S. and Baert, S. (in press), "The association between cultural distance and migrants' self-rated health", European Journal of Health Economics. DOI: 10.1007/s10198-017-0881-y.

Di Stasio, V. (2014), “Education as a Signal of Trainability: Results from a Vignette Study with Italian Employers", European Sociological Review, Vol. 30 No. 6, pp. 796-809.

Drydakis, N. (2014), "Sexual orientation discrimination in the Cypriot labour market. Distastes or uncertainty?", International Journal of Manpower, Vol. 35 No. 5, pp. 720-744.

Drydakis, N. (2015), “Measuring Sexual Orientation Discrimination in the UK's Labour Market; A Field Experiment", Human Relations, Vol. 68 No.11, pp. 1769-1796.

Drydakis, N. (2017), "Trans employees, transitioning, and job satisfaction", Journal of Vocational Behavior, Vol. 98 No. 1, pp. 1-16.

FRA (2014), "Being Trans in the European Union. Comparative analysis of EU LGBT survey data", European Union Agency for Fundamental Rights, Vienna.

Geijtenbeek, L. and Plug, E. (2015), "Is There a Penalty for Becoming a Woman? Is There a Premium for Becoming a Man? Evidence from a Sample of Transgender Workers", IZA Discussion Paper Series [9077], Institute for Labor Economics, Bonn. 
Grant, J. M., Mottet, L., Tanis, J. E., Harrison, J., Herman, J. and Keisling, M. (2011), "Injustice at Every Turn: A report of the National Transgender Discrimination Survey", National Center for Transgender Equality, Washington DC.

Heilman, M. E. (1983), "Sex bias in work settings: The lack of fit model", In Staw, B. and Cummings, L. (Eds.), Research in Organizational Behavior Vol.5, JAI Press, Greenwich, CT, pp. 269-298.

Hill, D. B. and Willoughby, B. L. (2005), "The development and validation of the genderism and transphobia scale", Sex Roles, Vol. 53 No.7-8, pp.531-544.

Hughto, J. M. W., Reisner, S. L. and Pachankis, J. E. (2015), "Transgender stigma and health: a critical review of stigma determinants, mechanisms, and interventions", Social Science \& Medicine, Vol. 147 No. 12, pp. 222-231.

ILGA-Europe (2017), "Annual Review of the Human Rights Situation of Lesbian, Gay, Bisexual, Trans and Intersex People in Europe", ILGA-Europe, Brussels.

James, S. E., Herman, J. L., Rankin, S., Keisling, M., Mottet, L. and Anafi, M. (2016), “The report of the 2015 US transgender survey", National Center for Transgender Equality, Washington DC.

Kerns, J. G. and Fine, M. A. (1994), "The relation between gender and negative attitudes toward gay men and lesbians: Do gender role attitudes mediate this relation?", Sex Roles, Vol. 31 No. 5-6, pp. 297-307.

Make the Road New York. (2010), "Transgender need not apply: A report on gender identity job discrimination", Make the Road New York, New York.

Motmans, J., de Boilley, I. and Debunne, S. (2009), "Leven als transgender in België: de sociale en juridische situatie van transgender personen in kaart gebracht", Instituut voor de gelijkheid van vouwen en mannen, Brussel.

Patacchini, E., Ragusa, G. and Zenou, Y. (2015), "Unexplored dimensions of discrimination in Europe: homosexuality and physical appearance", Journal of Population Economics, Vol.28 No.4, pp. 1045-1073.

Reynolds, W. M. (1982), "Development of reliable and valid short forms of the MarloweCrowne Social Desirability Scale", Journal of Clinical Psychology, Vol. 38 No. 1, pp. 119-125. 
Sârbescu, P., Costea, I. and Rusu, S. (2011), "Psychometric properties of the MarloweCrowne Social Desirability Scale in a Romanian sample", Procedia-Social and Behavioral Sciences, Vol. 33 No. 1, pp. 707-711.

Schilt, K. and Wiswall, M. (2008), "Before and after: Gender transitions, human capital, and workplace experiences" BE Journal of Economic Analysis \& Policy, Vol. 8 No. 1, pp. 39.

Stone, A. L. (2009), "More than adding a T: American lesbian and gay activists' attitudes towards transgender inclusion" Sexualities, Vol. 12 No. 3, pp. 334-354.

Valfort, M. (2017), "LGBTI in OECD Countries", OECD Publishing, Paris.

Van Belle, E., Caers, R., De Couck, M., Di Stasio, V. and Baert, S. (2017), "Why Is unemployment duration a sorting criterion in hiring?", IZA Discussion Paper Series [10876], Institute of Labor Economics, Bonn, July 2017.

Van Hoye, G. and Lievens, F. (2003), "The Effects of Sexual Orientation or Hirability Ratings: An Experimental Study", Journal of Business and Psychology, Vol. 18 No. 1, pp. 1530.

Weichselbaumer, D. (2015), "Testing for Discrimination against Lesbians of Different Marital Status: A Field Experiment", Industrial Relations, Vol. 54 No. 1, pp. 131-161.

West, K. and Hewstone, M. (2012), "Culture and contact in the promotion and reduction of anti-gay prejudice: Evidence from Jamaica and Britain", Journal of Homosexuality, Vol. 59 No. 1, pp. 44-66. 
Figure 1. Mediation Model

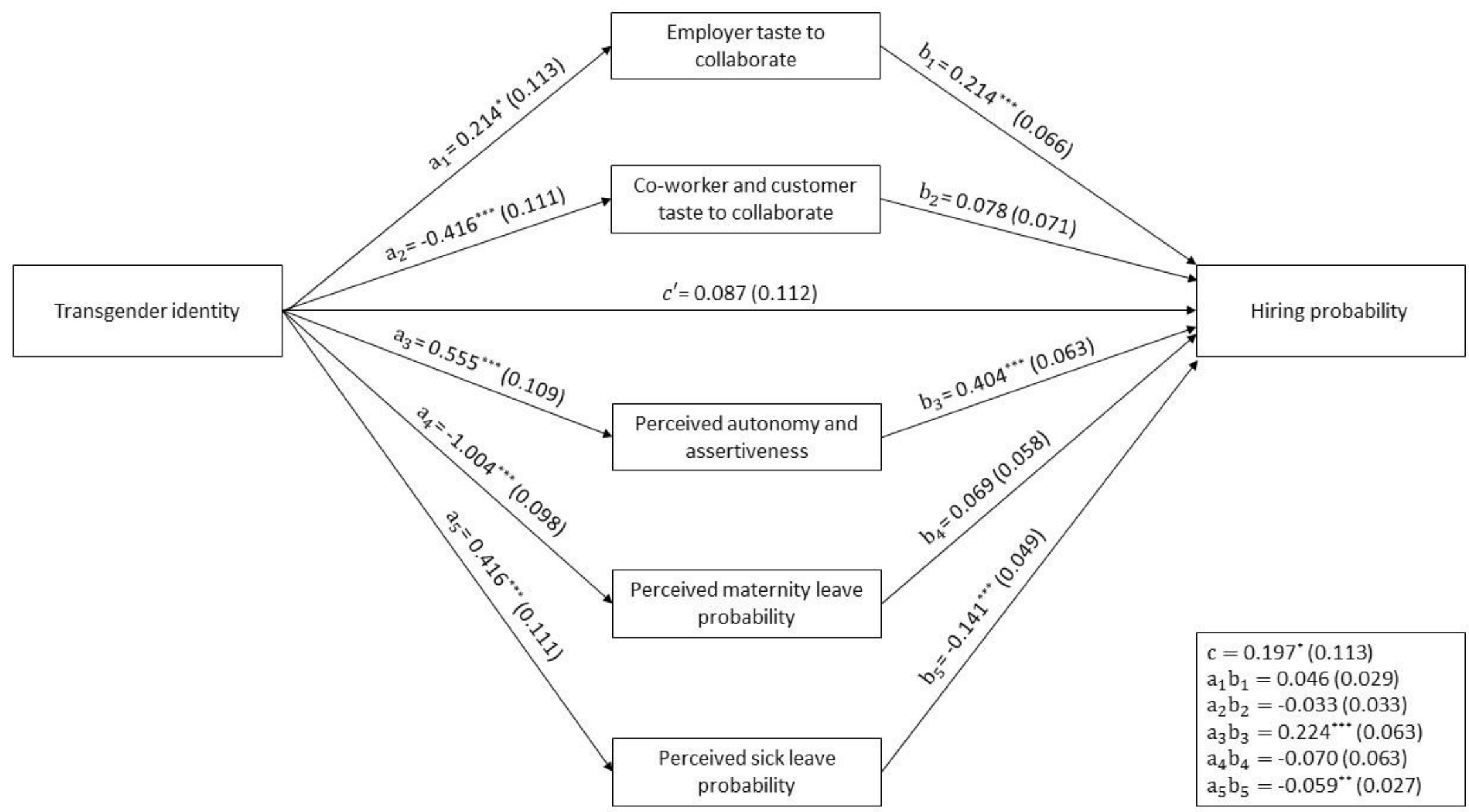

Notes. The presented statistics are coefficient estimates and standard errors in parentheses for the mediation model outlined in Section 3.1. $c$ stands for the total association, $c^{\prime}$ for the direct association, and $a_{i} b_{i}$ for the indirect associations between the transgender identity of the candidate and her hiring probability. The standard errors for the indirect associations are based on 10,000 bootstrap samples. ${ }^{* * *}\left({ }^{* *}\right)\left(\left({ }^{*}\right)\right)$ indicates significance at the $1 \%(5 \%)((10 \%))$ significance level. 


\begin{tabular}{|c|c|c|c|}
\hline & (1) & $(2)$ & (3) \\
\hline & \multicolumn{2}{|l|}{ Mean } & \multirow[b]{2}{*}{ Difference: $(2)-(1)$} \\
\hline & $\begin{array}{l}\text { Control candidate } \\
N=155\end{array}$ & $\begin{array}{l}\text { Transgender candidate } \\
\mathrm{N}=155\end{array}$ & \\
\hline \multicolumn{4}{|l|}{ A. Manipulation check } \\
\hline 'The candidate was born as a woman' & 6.287 & 1.619 & $-4.668^{* * *}[30.232]$ \\
\hline \multicolumn{4}{|l|}{ B. Occupation } \\
\hline Management assistant & 0.335 & 0.329 & $-0.006[0.120]$ \\
\hline Logistics clerk & 0.342 & 0.335 & $-0.006[0.120]$ \\
\hline Site manager & 0.323 & 0.335 & $0.013[0.241]$ \\
\hline \multicolumn{4}{|l|}{ C. Participant characteristics } \\
\hline Male gender & 0.195 & 0.201 & $0.006[0.143]$ \\
\hline Age & 22.591 & 22.305 & $-0.286[1.221]$ \\
\hline Foreign nationality & 0.169 & 0.130 & $-0.039[0.958]$ \\
\hline Father with tertiary education & 0.592 & 0.526 & $-0.066[1.164]$ \\
\hline Mother with tertiary education & 0.662 & 0.578 & $-0.084[1.527]$ \\
\hline Lesbian, gay, or bisexual sexual orientation & 0.052 & 0.058 & $0.006[0.195]$ \\
\hline Conformity to classical gender roles scale & 1.901 & 1.922 & $0.021[0.313]$ \\
\hline Contact with transgenders scale & 1.377 & 1.403 & $0.027[0.351]$ \\
\hline Social desirability bias scale & 0.558 & 0.557 & $-0.001[0.046]$ \\
\hline \multicolumn{4}{|l|}{ D. Candidate evaluation scores } \\
\hline 'I will invite the candidate for a job interview' & 5.887 & 6.068 & $0.181[1.515]$ \\
\hline 'I will hire the job candidate' & 4.900 & 5.113 & $0.213^{*}[1.737]$ \\
\hline 'As an employer, I will enjoy collaborating with this candidate' & 5.019 & 5.232 & $0.213^{*}[1.888]$ \\
\hline 'My co-workers will enjoy collaborating with this candidate' & 4.861 & 4.406 & $-0.455^{* * *}[3.958]$ \\
\hline 'My customers will enjoy collaborating with this candidate' & 4.903 & 4.532 & $-0.371^{* * *}[3.025]$ \\
\hline 'This candidate will provide sufficient autonomy' & 5.210 & 5.671 & $0.461^{* * *}[4.011]$ \\
\hline 'This candidate will provide sufficient assertiveness' & 4.852 & 5.461 & $0.610^{* * *}[5.050]$ \\
\hline 'This candidate will be on maternity leave in the short or medium run' & 3.752 & 2.123 & $-1.629^{* * *}[10.204]$ \\
\hline 'This candidate will be on sick leave in the short or medium run' & 2.619 & 3.126 & $0.506^{* * *}[3.737]$ \\
\hline
\end{tabular}

Notes. See Section 2.1 for a description of the participant characteristics scales. All candidate evaluation statements are scored on a 7-point Likert scale. T-tests are performed to test whether the differences presented in Column (3) are significantly different from $0 .{ }^{* * *}\left({ }^{* *}\right)\left(\left(^{*}\right)\right)$ indicates significance at the $1 \%(5 \%)((10 \%))$ significance level. T-statistics are between brackets. 
1 UNESCO (2016) defines a transgender woman (man) as a person who was registered as a boy (girl) at birth but has a female (male) gender identity up to date.

2 This might be related to the irreversible sterilisation that is undergone in certain medical procedures (De Sutter, 2001).

3 In addition, subjective measures of discrimination against transgender individuals are presented in Brewster et al. (2012) and Brewster et al. (2014).

4 The PEAF has an indication of the occupational aspirations of all unemployed in their database. More concretely, each unemployed indicates up to eight occupations, according to PEAF's classification system, in which they are interested. By September 2012, 91.1\% (47.4\%) ((13.8\%)) of the unemployed willing to work as a management assistant (logistics clerk) ((site manager)) were female.

${ }^{5}$ Source: http://transgenderinfo.be/.

${ }^{6}$ Moreover, due to the change in gender recognition legislation of January 2018 in Flanders (mentioned in Section 2.2), Flemish organisations guiding transgender individuals may advise them to disclose their transgender identity in another way than was the case in our experiment, with further consequences for the external validity of our experiment. 